Corrigendum: Genome-wide association study identifies variants at $C L U$ and PICALM associated with Alzheimer's disease

Denise Harold, Richard Abraham, Paul Hollingworth, Rebecca Sims, Amy Gerrish, Marian L Hamshere, Jaspreet Singh Pahwa, Valentina Moskvina, Kimberley Dowzell, Amy Williams, Nicola Jones, Charlene Thomas, Alexandra Stretton, Angharad R Morgan, Simon Lovestone, John Powell, Petroula Proitsi, Michelle K Lupton, Carol Brayne, David C Rubinsztein, Michael Gill, Brian Lawlor, Aoibhinn Lynch, Kevin Morgan, Kristelle S Brown, Peter A Passmore, David Craig, Bernadette McGuinness, Stephen Todd, Clive Holmes, David Mann, A David Smith, Seth Love, Patrick G Kehoe, John Hardy, Simon Mead, Nick Fox, Martin Rossor, John Collinge, Wolfgang Maier, Frank Jessen, Britta Schürmann, Hendrik van den Bussche, Isabella Heuser, Johannes Kornhuber, Jens Wiltfang, Martin Dichgans, Lutz Frölich, Harald Hampel, Michael Hüll, Dan Rujescu, Alison M Goate, John S K Kauwe, Carlos Cruchaga, Petra Nowotny, John C Morris, Kevin Mayo, Kristel Sleegers, Karolien Bettens, Sebastiaan Engelborghs, Peter P De Deyn, Christine Van Broeckhoven, Gill Livingston, Nicholas J Bass, Hugh Gurling, Andrew McQuillin, Rhian Gwilliam, Panagiotis Deloukas, Ammar Al-Chalabi, Christopher E Shaw, Magda Tsolaki, Andrew B Singleton, Rita Guerreiro, Thomas W Mühleisen, Markus M Nöthen, Susanne Moebus, Karl-Heinz Jöckel, Norman Klopp, H-Erich Wichmann, Minerva M Carrasquillo, V Shane Pankratz, Steven G Younkin, Peter A Holmans, Michael O’Donovan, Michael J Owen \& Julie Williams

Nat. Genet. 41, 1088-1093 (2009); published online 6 September 2009; corrected after print 9 May 2013

In the version of this article initially published, Reinhard Heun was not included in the author list. This has been corrected in the HTML and PDF versions of the article.

\title{
Corrigendum: Genome-wide meta-analyses of multiancestry cohorts identify multiple new susceptibility loci for refractive error and myopia
}

Virginie J M Verhoeven, Pirro G Hysi, Robert Wojciechowski, Qiao Fan, Jeremy A Guggenheim, René Höhn, Stuart MacGregor, Alex W Hewitt, Abhishek Nag, Ching-Yu Cheng, Ekaterina Yonova-Doing, Xin Zhou, M Kamran Ikram, Gabriëlle H S Buitendijk, George McMahon, John P Kemp, Beate St Pourcain, Claire L Simpson, Kari-Matti Mäkelä, Terho Lehtimäki, Mika Kähönen, Andrew D Paterson, S Mohsen Hosseini, Hoi Suen Wong, Liang Xu, Jost B Jonas, Olavi Pärssinen, Juho Wedenoja, Shea Ping Yip, Daniel W H Ho, Chi Pui Pang, Li Jia Chen, Kathryn P Burdon, Jamie E Craig, Barbara E K Klein, Ronald Klein, Toomas Haller, Andres Metspalu, Chiea-Chuen Khor, E-Shyong Tai, Tin Aung, Eranga Vithana, Wan-Ting Tay, Veluchamy A Barathi, Consortium for Refractive Error and Myopia (CREAM), Peng Chen, Ruoying Li, Jiemin Liao, Yingfeng Zheng, Rick T Ong, Angela Döring, The Diabetes Control and Complications Trial/Epidemiology of Diabetes Interventions and Complications (DCCT/EDIC) Research Group, David M Evans, Nicholas J Timpson, Annemieke J M H Verkerk, Thomas Meitinger, Olli Raitakari, Felicia Hawthorne, Tim D Spector, Lennart C Karssen, Mario Pirastu, Federico Murgia, Wei Ang, Wellcome Trust Case Control Consortium (WTCCC), Aniket Mishra, Grant W Montgomery, Craig E Pennell, Phillippa M Cumberland, Ioana Cotlarciuc, Paul Mitchell, Jie Jin Wang, Maria Schache, Sarayut Janmahasathian, Robert P Igo Jr, Jonathan H Lass, Emily Chew, Sudha KIyengar, The Fuchs' Genetics MultiCenter Study Group, Theo G M F Gorgels, Igor Rudan, Caroline Hayward, Alan F Wright, Ozren Polasek, Zoran Vatavuk, James F Wilson, Brian Fleck, Tanja Zeller, Alireza Mirshahi, Christian Müller, André G Uitterlinden, Fernando Rivadeneira, Johannes R Vingerling, Albert Hofman, Ben A Oostra, Najaf Amin, Arthur A B Bergen, Yik-Ying Teo, Jugnoo S Rahi, Veronique Vitart, Cathy Williams, Paul N Baird, Tien-Yin Wong, Konrad Oexle, Norbert Pfeiffer, David A Mackey, Terri L Young, Cornelia M van Duijn, Seang-Mei Saw, Joan E Bailey-Wilson, Dwight Stambolian, Caroline C Klaver \& Christopher J Hammond Nat. Genet. 45, 314-318 (2013); doi:10.1038/ng.2554; published online 10 February 2013; corrected after print 9 May 2013

In the version of this article initially published, the affiliations of Daniel W.H. Ho were incorrect, and the spelling of Sarayut Janmahasatian in the author list was incorrect. The errors have been corrected in the HTML and PDF versions of this article. 